Wertewandel als Grundlage nachhaltiger Raumentwicklung

\title{
Alter Wein in neuen Schläuchen?
}

\author{
Nachhaltige Entwicklung erfordert einen neuen gesellschaftlichen Konsens über \\ Werte in Bezug auf das praktische Umgehen mit der Mitwelt. Die Anwendung \\ dieser Werte auf den Bereich der Raumentwicklung macht klar, dass kosme- \\ tische Verbesserungen des bestehenden Systems nicht ausreichen, um nachhal- \\ tige Entwicklung zu erreichen. Diese erfordert eine grundlegende Neustruktu- \\ rierung der Aushandlung raumwirksamer Problemstellungen.
}

\section{$\mathrm{W}$}

\section{Von Michael Narodoslawsky} ie bei jedem wichtigen gesellschaftlichkulturellen Veränderungsprozess ranken sich auch um die Entstehung des Konzeptes nachhaltiger Entwicklung eine Reihe von Mythen. Ein solcher Mythos betrifft - zumindest im deutschen Sprachraum - die Herkunft des Begriffes Nachhaltigkeit selbst: Er soll, so wird uns erklärt, aus der Forstwirtschaft abgeleitet werden, wo man schon vor fast zweihundert Jahren erkannt hat, dass wenn man mehr erntet als nachwächst zum Schluss kein Wald mehr übrig bleibt.

Ähnliche Mythen der Vater- und Mutterschaft ranken sich natürlich auch um andere Bereiche, vom Umwelt- und Naturschutz bis zu den sozialen Konzepten der 68er Generation. Ein solcher Mythos rankt sich auch um die Raumordnung. Auch in diesem Bereich wird durchaus der Anspruch erhoben, Nachhaltigkeit bereits seit vielen Jahren zu praktizieren. Raumordnung hat ja das Ziel, die räumlich bedingten Unterschiede in den sozialen und wirtschaftlichen Chancen auszugleichen. Sie sei damit nichts anderes als ein Ausdruck der vielzitierten sozialen und wirtschaftlichen Dimension nachhaltiger Entwicklung. Raumordnung soll räumliche Nutzungskonflikte lösen und damit Naturraum sichern bei gleichzeitiger Erfüllung der wirtschaftlichen und sozialen Erfordernisse der gesellschaftlichen Entwicklung. Konzepte wie dezentrale Zentren und endogene Regionalentwicklung seien schon in der Raumplanung diskutiert worden, lange bevor die regionale Komponente nachhaltiger Entwicklung erkannt wurde. Aus dieser Sichtweise ist nachhaltige Entwicklung für die Raumordnung nicht nur nichts Neues. Sie ist nichts anderes als die Anwendung der Grundsätze der Raumordnung auf andere gesellschaftliche Bereiche.

Diese Mythen sind - wie alle Mythen - nicht so sehr Ausdruck einer geschichtlichen Wahrheit als vielmehr ein Hinweis auf den Erfolg des Konzeptes nachhaltiger Entwicklung. Man könnte über diese Mythenbildung lächelnd hinweg gehen, wenn sie nicht auch eine gewisse Gefahr darstellen würde. Die Gefahr ist, dass sich solche Mythen trefflich als Vorwand nichts zu tun verwenden lassen. Denn ob nun der Begriff Nachhaltigkeit aus der Forstwirtschaft kommt oder nicht, die heutige Forstwirtschaft mit ihrem massiven Einsatz fossiler Rohstoffe, hauptsächlich als Kraftstoff für die immer größer werdenden Ernte- und Rückmaschinen, entspricht nicht den Anforderungen nachhaltiger Entwicklung! Und ob die moderne Raumordnung nachhaltig ist, das mag jeder auf dem Parkplatz eines Mega-Einkaufszentrums am Rande einer Stadt selbst entscheiden.

Dieser Beitrag will aber nicht die Mythen nachhaltiger Entwicklung entzaubern. Es soll vielmehr dargestellt werden, dass Raumentwicklung ein zentrales Thema nachhaltiger Entwicklung ist, und dass nachhaltige Raumentwicklung einen Bruch mit der Tradition konventioneller Raumordnung darstellt. Dieser Bruch gründet sich auf einem tiefgreifenden Wandel gesellschaftlicher Werte.

\section{Ein Wertewandel - woher und wohin?}

In den letzten Jahren taucht in der Diskussion über nachhaltige Entwicklung immer wieder die Frage eines Wandels gesellschaftlicher Werte auf. Diese Diskussion ist noch durchaus unklar und diffus. Es ist noch nicht einmal ganz klar, ob nachhaltige Entwicklung die Folge eines Wertewandels darstellt oder ob nachhaltige Entwicklung einen solchen Wertewandel zu ihrer Durchsetzung braucht. Schließlich ist die Diskussion auch insofern noch im Anfangsstadium, als es keinen Konsens darüber gibt, wie denn etwa Werte für eine nachhaltige Entwicklung aussehen müssten.
Im Angesicht des herrschenden Wertepluralismus erscheint es sogar einigermaßen frivol, nachhaltige Entwicklung mit einem allgemeinen Wandel der Werte in Zusammenhang zu bringen. $\mathrm{Zu}$ weit klaffen die Wertesysteme in unserer Gesellschaft auseinander, zu stark erscheint die Zentrifugalkraft, die diese Wertesysteme immer weiter auseinander treibt, um in absehbarer Zukunft auf eine Kongruenz der Werte zu hoffen, die auch noch in Übereinstimmung mit nachhaltiger Entwicklung stehen müssten.

Vor diesem Hintergrund fand im Dezember 2001 das Symposium ,,Societal Values for Sustainable Development“" in Seggau/Österreich statt (1). Die Ergebnisse der Diskussionen auf diesem Symposium sind auch für das Thema Raumentwicklung und Nachhaltigkeit von Interesse und sollen daher hier kurz dargestellt werden:

Es ist tatsächlich so, dass gesellschaftliche Wertesysteme immer diverser werden. Ein alles umgreifendes Wertesystem, das noch dazu auch global, über verschiedene Kulturen hinweg gültig ist, ist nicht absehbar. Nachhaltige Entwicklung erfordert aber keineswegs einen vollständigen Konsens über alle Werte. Obwohl nachhaltige Entwicklung heute als das umfassendste und weitreichendste Entwicklungskonzept angesehen werden muss, so betrifft es doch nur einen eingeschränkten Bereich eines gesellschaftlichen Wertesystems: Es geht um jene Werte, die den praktischen Umgang mit der Mitwelt, also Gesellschaft und Natur regeln. Weite und kontroverse Teile von Wertesystemen, etwa jene, die die Zielsetzung der individuellen oder gesellschaftlichen Entwicklung betreffen, sind für die Umsetzung nachhaltiger Entwicklung kaum relevant.

In dem beschränkten Feld der Werte, das für nachhaltige Entwicklung relevant ist, zeichnet sich sehr wohl ein praktischer Konsens ab. Einige Wertekategorien, auf die wir noch eingehen werden, finden tatsächlich immer stärkere Unterstïtzung quer durch Wertesysteme und Kulturen. Dies ist ähnlich zu sehen wie der Wertekanon der allgemeinen Menschenrechte, die, obwohl eindeutig aus westlich-aufklärerischer Quelle kommend, auch in andere Kulturen und in fast alle gesellschaftlichen Wertesysteme Eingang gefunden haben.

\section{Drei zentrale Werte}

Wir wollen uns nun der inhaltlichen Seite dieser Werte-Diskussion zuwenden. Obwohl es noch keineswegs einen allgemein anerkannten „Nachhaltigkeits-Kanon“ gibt, so sind doch einige Kon- 
turen in dieser inhaltlichen Diskussion über Werte für nachhaltige Entwicklung absehbar. Die für unsere Themenstellung Wichtigsten sind:

- Die Bewertung von Vernetzung: Vernetzung ist in gewisser Weise zu einem Modewort unserer Zeit geworden. Die Tätigkeit des Vernetzens hat einen eigenen positiven Wert angenommen, vernetzt sein ist besser als isoliert bleiben. Vernetzung wird als eine notwendige Grundvoraussetzung der Entwicklung angesehen, isolierten Systemen wird immer mehr die Entwicklungsfähigkeit abgesprochen. Dies gilt auch für das Wechselspiel zwischen Natur und Mensch. Die Natur wird immer mehr als Partner in einem komplizierten Netzwerk der Wechselwirkungen und damit als Partner der Gesellschaft gesehen.

Das In-Wert-Setzen anderer Systeme. Dies ist eine Folge der Vernetzung. Vernetzung erfordert die Anerkennung des Eigenwertes der Partner im Netz, sie erfordert die Erkenntnis, dass das ganze Netz aus wertvollen Partnern besteht, deren Integrität erhalten werden muss. Dies gilt für die gesamte Mitwelt, also auch für die Natur.

- Der Vorrang von Entwicklung vor Statik. Vernetzung zwischen wertvollen Partnern ist nur dann aufrecht zu erhalten, wenn alle Partner innerhalb des Netzes sich positiv entwickeln können. Vernetzung ist grundsätzlich ein dynamischer Vorgang, jeder Teilnehmer behält ja seine Integrität. Das Netz selbst ist für alle Partner ein weiterer Freiheitsgrad in ihrer Entwicklung und ist selbst eine sich entwickelnde Einheit. Bricht die Entwicklung ab, so stirbt das Netz und jeder Partner im Netz ist um eine Entwicklungschance ärmer.

Diesem einigermaßen einfachen Wertekodex wird heute kaum mehr widersprochen. Aus ihm heraus lässt sich der gesamte Bogen nachhaltiger Entwicklung aufspannen. Wir wollen nun kurz diskutieren, was diese Wertegrundlage für nachhaltige Raumentwicklung bedeutet.

\section{- Nachhaltigkeits-Werte und Raumentwicklung}

Nachhaltige Entwicklung betrifft das Zusammenspiel zwischen ökologischen und gesellschaftlichen Prozessen. All diese Prozesse haben naturgemäß ihren eigenen räumlichen Bezug, ihre eigene räumliche Dimension. Kaum ein anderer Bereich ist daher für das Schicksal nachhaltiger Entwicklung so entscheidend wie die Raumentwicklung.

Folgt nachhaltige Entwicklung dem hier vorgestellten Wertekanon, so ergeben sich aber wesentliche Abweichungen zur bisherigen Praxis der
Raumordnung und Raumplanung. Dies beginnt schon bei der Begrifflichkeit: Man spricht bisher von Raumordnung und Raumplanung. Das sind statische Begriffe, die eigentlich einer Entwicklung, noch dazu einer Entwicklung von eigenständigen Partnern in einem vernetzten System entgegenstehen. Entwicklung ist grundsätzlich ein offener Prozess, Planung ist immer mit einem Abschluss und Ziel verbunden. Der hier dargestellte Wertekanon erfordert daher rein begrifflich den Übergang von Raumplanung zu Raumentwicklung.

Das ist keineswegs nur Wortspielerei. Es erfordert eine grundsätzliche Umorientierung, für die wir derzeit nur schlecht gerüstet sind. Entwicklung ist ein ständiger Aushandlungsprozess zwischen allen Betroffenen, Planung ist ein einmaliger Entscheidungsprozess durch eine höhere Instanz. Planung ist inhärent mit Hierarchie verbunden, Entwicklung mit Partizipation.

Hier ergibt sich eine erste Herausforderung durch das Ziel einer nachhaltigen Entwicklung, die das bestehende System der politischen Hierarchien, auf denen die konventionelle Raumplanung basiert, nicht erfüllen kann: Unsere Aktivitäten haben sehr unterschiedliche Raumbezüge. Ein Kraftwerk etwa beeinflusst mit seinen Kohlendioxidemissionen das globale Klimasystem, mit seinen Stickoxidemissionen regionale Ökosysteme. Sein Brennstoff kommt aus dem Mittleren Osten und verursacht bei seiner Gewinnung dort soziale und ökologische Probleme. Unser Verbrauch wird über ein kontinentales Netz (sic!) befriedigt, durch das Emission und Konsum entkoppelt werden.

Es ist eine Tatsache, dass uns die Institutionen ganz zu schweigen von der Konfliktlösungskultur - für die Aushandlung derartiger Raumnutzungsprobleme fehlen. Es geht daher nicht um eine neue und optimierte Art der Raumplanung. Es geht um eine neue Grundlage unserer politischen Aushandlung vor dem Hintergrund raumbezogener Probleme!

Ein ähnliches Problem ergibt sich aus der Forderung des In-Wert-Setzens anderer, vor allem natürlicher, Systeme. Bisher war, auch in der Raumplanung, Natur zum Nulltarif verfügbar. Erst durch ihre Nutzung durch den Menschen erhielten natürliche Ressourcen ihren Wert. Für sich selbst gesehen waren sie wertlos. Wie können wir von einem Plünderungstarif für natürliche Systeme zu einem Wertsystem gelangen, das die Entwicklungsmöglichkeit von ökologischen Systemen widerspiegelt?
Auch hier stehen wir vor einem grundlegenden Problem. Alle bisherigen Versuche, Natur mit unserem monetären Wertsystem zu messen, scheitern letztlich daran, dass wir immer nur unser eigenes Wertsystem auf die Natur projizieren. Auch hier haben wir weder die Institutionen noch die Methoden verfügbar, um die Erfordernisse der Evolution natürlicher Systeme in die Aushandlung unserer Raumnutzungsansprïche aufzunehmen. Was wir aber nicht in diesen Prozess der Aushandlung aufnehmen können, wird schlussendlich zu kurz kommen und damit das Funktionieren des Gesamtsystems in Frage stellen.

\section{- Never Wein - aber wo sind die Schläuche?}

Ein neuer gesellschaftlicher Konsens über Werte in Bezug auf das praktische Umgehen mit der Mitwelt und nachhaltige Entwicklung gehen Hand in Hand. Die Anwendung dieser Werte auf den Bereich der Raumentwicklung macht aber klar, dass kosmetische Verbesserungen des bestehenden Systems nicht ausreichen, um nachhaltige Entwicklung zu erreichen. Nachhaltige Entwicklung erfordert vielmehr eine grundlegende Neustrukturierung der Aushandlung raumwirksamer Problemstellungen. Diese Neustrukturierung partizipativer Governance-Systeme wird zu einer Hauptherausforderung der Umsetzung nachhaltiger Entwicklung.

\section{Anmerkungen}

(1) Symposiumsunterlagen sind über die Homepage von ENSURE unter www.european-association.org/ensure abrufbar.

\section{Der Autor}

Dr. Michael Narodoslawsky ist Professor an der Technischen Universität Graz und Vorsitzender des Executive Committee von ENSURE (European Network of Sustainable Urban and Regional Development. Kontakt: Institut für Grundlagen der Verfahrenstechnik und Anlagentechnik, TU Graz, A-8010 Graz, Inffeldgasse 25. Tel. 0043-316-873-7465,

E-Mail: braunegg@glvt.tu-graz.ac.at 
(c) 20I0 Authors; licensee IÖW and oekom verlag. This is an article distributed under the terms of the Creative Commons Attribution Non-Commercial No Derivates License (http://creativecommons.org/licenses/by-nc-nd/3.o/), which permits unrestricted use, distribution, and reproduction in any medium, provided the original work is properly cited. 